\title{
Experiments with Filaments Heated Electrically in Volatile Liquids
}

This content has been downloaded from IOPscience. Please scroll down to see the full text. 1915 Proc. Phys. Soc. London 2851

(http://iopscience.iop.org/1478-7814/28/1/306)

View the table of contents for this issue, or go to the journal homepage for more

Download details:

IP Address: 141.161.91.14

This content was downloaded on 08/09/2015 at 17:33

Please note that terms and conditions apply. 
VI. Experiments uith Filaments Heaten Electrically in Volatile Liquids. By S. W. J. Sмттн, Hon. Sec. Plıysical Socirty.

Received September 24, 1915. Read May 22, 1914.

1. I was led to perform these experiments by a statement: made to me hy Mr. C. W. S. Cruwley* to the effect that, when a 100 volt lamip filled with paraffin oil is used as a resistance in a 200 volt circuit, the bubbles of gas which form on the filament, instead of rising at once, run down the legs of the filament before they escape to the surface of the liquid.

Attempting to reproduce this phenomenon, I found that if the voltage applied to the lamp were reduced below 100 , so that no bubbles formed, it was possible (by momentarily increasing the voltage) to obtain a single bubble upon the wire.

The behaviour of this bubble was most fascinating to watch, and seemed even more mysterious than the observation to which my attention had been drawn.

The bubble ran backwards and forwards from one terminal to the other, "looping the loops" of the filament many times before escaping from the wire!

2. Many other liquids, besides paraffin oil, yield the same result. Benzene, turpentine and aniline may be mentioned as examples.

The currents necessary for the production of the bubble and for its maintenance are higher, the higher the boiling point of the liquid. This suggests that the bubble consists of vapour of the liquid, and the inference is, in fact, usually correct except

* Note by Mr. Crawley.- " The effect in question was shown to me by Mr. Addenbrooke, who was, however, too much occupied to bring it forward himself. The "pip' of an ordinary incandescent lamp had been broken off and the bulb filled with paraffin oil, for use as a resistance. It was found most satisfactory both as being able to absorb more power than with a vacuum and also to stand momentary overload much better, and for this reason alone it is worth mentioning.

"When the current is flowing there is naturally a strong current of hot oil up the legs of the filament. When sufficient current is used bubbles form on the filament, but these, instead of rising to the surface, run down the legs against both gravity and the upward current of hot oil and come off at the bottom. Having no facilities for investigating the phenomenon, I thought it might be worth while to draw attention to it in the hope that some member of the Society might be tempted to study it. Meanwhile, Dr. Smith has kindly looked into it and carried the matter further and, it seems to me, given the true explanation."-May 27, 1914. 
during the first heatings soon after the liquid is poured into the lamp. Then there will be a certain amount of entrapped air which may provide the nuclei of the earlier bubbles. Indeed, if a very viscous liquid of high boiling point (such as machine oil) is used it is possible by means of a fine pipette to project a minute bubble of air upon the heated wire. This bubble then moves in the same manner as those formed spontaneously.*

3. Under favourable circumstances, with turpentine for example, it is easy to adjust the current so that a fairly large bubble circulates to and fro for a long time (a quarter of an hour, for instance) without appreciable change in size. Relucing the current a similar steady state can be reached with a bubble of reduced size. If the current be reduced below a certain limit, however, the bubble disappears.

These facts suggest that the contents of the bubble are continually changing, the constant size under any particular gradient being the result of a balance between the increase clue to evaporation into the bubble and decrease due to condensation from it.

4. For, assuming as a rough approximation that the bubble is spherical, consider the effect of supposing its temperature to be uniform. The excess pressure within it will be given by $p r=Q \sigma$, where $r$ is the radius and $\sigma$ is the surface tension, at temperature 0 , of the bubble. Since $p$ falls and $\sigma$ rises when the temperature is lowered, it follows that the equilibrium value of $r$ would be increased by lowering $\theta$. But the bubble actually becomes smaller when the temperature is reduced. We must, therefore, conclude that its temperature is not uniform. The gradient from the wire nust be such that, although the hottest part of the smaller bubble is colder than that of the larger one, its mean temperature is above that of the larger one.

For a restricted range it is possible that when the temperature of the wire is reduced the mean temperature of the diminishing bubble may rise; but so soon as this becomes impossible the bubble must collapse, as experience shows.

It will also be noted that a spherical bubble of uniform temperature would be unstable under the conditions of the experiment.

To sum up, it appears from a variety of considerations that

* I have to thank my colleague, MIr, H. Moss, M.Sc., for kindly undertaking to perlorm this experiment. 
the bubble exists in a region of which the temperature changes rapidly in the direction perpendicular to the wire.

5 . At first sight it seems possible that the movements of the bubble are due to electrical forces; but, except that these may play some part in causing the bubble to cling to the wire, it is unlikely that they are appreciable.

The motion of the bubble is not affected by altering the direction of the current. It is produced equally well by alternating currents. It is thus apparently of thermal origin. and seems to be determined only by the temperatures to which the current is capable of raising the wire and its environment.

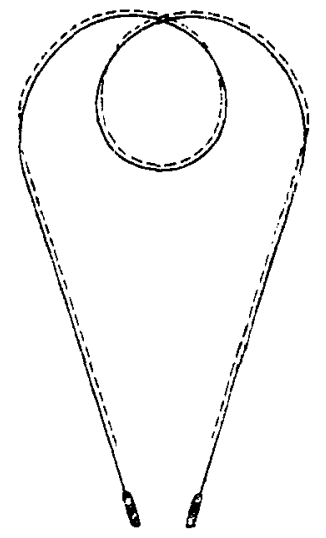

For convenience, the attempt to explain what is observed may be divided into two parts. Thus we may inquire why a moving bubble should follow the curvature of the wire before attempting to fix the cause of its motion.

6. A reason why a small rising bubble should follow the bend of the filament is not far to seel.

Keeping the current as low as possible, so that the motion of the bubble is comparatively slow and the convection currents from the heated wire are not very pronounced, it is possible to observe fairly easily the track which the bubble follows.* This track is represented by the dotted curves in the figure, the continuous curves denoting the filament of the lamp. The bubble may be said to pass along the "upper" side of each element of the filament. In other words, it runs along the

* Observation is facilitated by substituting a rectangular vessel, with plane glass sides, for the bulb of the lamp. 
region in which the convection of heated liquid from the filament is a minimum. It is easy to see how such a region arises - permitting a layer of relatively hot liquid to remain in contact with the wire. This " sheltered "layer will be hottest and therefore least viscous nearest the wire. The rate of increase of viscosity outwards will be greatest in a liquid for which the conductivity is small and the temperature coefficient of viscosity large. The bubble, moving along the wire, will be moving in a region in which the viscous resistance to the motion of that half of it which is nearer the wire is less than that experienced by the more distant half. Hence the bubble will be subject to a force tending to press it towards the wire, and this force will be greater the greater its velocity.

7. The above consideration supplies a partial explanation of the phenomenon, for it shows why a bubble may proceed for some distance round the bend of the filament with the impetus acquired during its rise.

Indeed, the necessity for a sufficient gradient of temperature near the wire, if the bubble is to follow it, can be shown in a very simple way.

With a liquid of low boiling point, such as ether, for example, it is only possible to obtain indications of the phenomenon under ordinary circumstances. Thus it is possible to obtain bubbles which will rise along the filament to its summit, and perhaps go a little way beyond, but it is impossible to make them descend very far. The buoyancy exceeds the force pressing the bubble towards the wire, and it escapes to the surface of the liquid.

But if a steeper temperature gradient from the wire to the liquid is made possible, by cooling the ether in a freezing mixture beforehand, it is easy to obtain bubbles which descend as in other liquids of higher boiling points.

('onversely if we take a liquid which shows the phenomenon under ordinary circumstances, we can cause its disappearance by raising the temperature of the liquid, sufficiently, beforehand.

8. Assuming that the variation of viscosity which accompanies the temperature gradient operates in the way indicated above, it remains to consider the origin of the moving force, other than gravity, which acts upon the bubble.

This force must obviously have a considerable component parallel to the wire, and this must act, at every instant, in the direction in which the bubble is moving. 
That this moving force depends upon the current strength, and, therefore, upon the temperature gradient, is easily shown.

Thus, if the current strength be slowly diminished, after the bubble has formed, the oscillations of the latter gradually diminish in amplitude until the motion extends over only a small arc near the summit of one of the limbs of the filament.

The moving force is now so small that a very small fraction, acting parallel to the wire, of the opposing force due to the buoyancy of the bubble is sufficient to bring it to rest.

9. In attempting to explain how the moving force arises, suppose, for simplicity, that the bubble is momentarily at rest upon a horizontal wire. It will be in a region where the successive filaments of liquid, above and parallel to the wire, are of decreasing temperature. These filaments will be in unstable equilibrium, the upper ones being heavier than the lower. The latter would rise through the former but for the presence of the wire.

Now imagine a small displacement of the bubble along the wire. This will tend to elevate the liquid immediately in front of the bubble and to depress that immediately behind it. To convey roughly what is meant, the case of a sphere moving along the bottom of a horizontal trough containing a layer of water, with a layer of oil above it, may be cited. If the trough has glass sides, the water can be seen to rise in front of the sphere whilst the oil falls in behind the sphere as it moves. This takes place although the oil is much less dense than the water and the surface tension between them is considerable. A similar but more pronounced effect may, therefore, be anticipated in the case under consicleration where the upper layers are denser than the lower and surface tension is inappreciable.

It is conceivable that this action once begun might continue of itself and keep the bubble moving forward. But, apart from this possibility, the colder liquid falling in behind the bubble will at once expand owing to its proximity to the wire and will thus tend to push the bubble forward in the direction in which it has begun to move.

The same kind of impulse will be produced in a similar way when the bubble is displaced downwards on a wire which is not horizontal and may be sufficient to enable it to move "against wravity."

In accordance with the facts, the distance through which the bubble is able to move in this way will depend upon the steepness of the temperature gradient, i.e., upon the current flowing through the lamp. 
The foot of each limb of the filament is comparatively cold owing to its nearness to the thick leading-in wire. Consequently the downward impulse will rapidly diminish as the bubble nears the end of the wire. The upward influence of gravity* will thus eventually assume predominance, bringing the bubble to rest and then starting it upon its return path.

10. According to the views just expressed, the bubble is continuously removing hotter layers of liquid from the neighbourhood of the wire and replacing them by colder ones. Consequently, in order to dissipate electric energy at a given rate, the temperature of the wire need not be so high when the bubble is present as when it is absent.

To fund whether the difference was easily perceptible some observations were made with a platinum filament immersed in turpentine.t $\mathrm{A}$ bubble having been formed upon the wire, simultaneous readings of the current through it and the voltage between the terminals were made, as the current was reduced, with the following results :--

\begin{tabular}{|c|c|}
\hline \multicolumn{2}{|c|}{ Bubble on wire. } \\
\hline Amperes. & Watts. \\
\hline $2 \cdot 5$ & $4 \cdot 25$ \\
$2 \cdot 4$ & $3 \cdot 87$ \\
$2 \cdot 3$ & $3 \cdot 4 !$ \\
$2 \cdot 2$ & $3 \cdot 18$ \\
\hline
\end{tabular}

The bubble disappeared when the current was lowered to 2.2 amperes. The current was then gradually raised to $2 \cdot 5$ anperes again (there being now no bubble present) and simultaneous readings of current and voltage were taken as before:

\begin{tabular}{ccc} 
& No bubble. & Watts. \\
\hline Amperes. & $4 \cdot 34$ \\
\hline $2 \cdot 5$ & $3 \cdot 9 \cdot 3$ \\
$2 \cdot 4$ & $335 \%$ \\
$2 \cdot 3$ & $3 \cdot 18$ \\
$2 \cdot 2$ & & \\
\hline
\end{tabular}

* In my experiments the upward currents of hot oil referred to by Mr. Crawley were inconspicuous, except near the vertical parts of the filament. $\dagger$ A large quantity of turpentine was used in order to reduce, as much as possible, fluctuations in the temperature of the liquid as a whole during the course of these observations. Check measurements were made to ensure, for example, that the differences shown in the Tables were not attributable solely to a gradual rise in temperature of the liquid during the observations. From their nature the data are not to be regarded as more than qualitatively correct. They may, moreover, indicate merely the effects of generil disturbanco (equivalent to stirring) produced by the moving bubble. 
From these data it will be seen that, when equal quantities of energy are being dissipated, the current is always greater, and therefore the resistance is always less, when the bubble is present than when it is absent. Since the wire is of platinum, the temperature must be less when the resistance is less.

If the bubble is watched it is seen to be followed by a trail of liquid, evidently of very different density from the main bulk, moving outwards from the part of the bubble furthest from the wire. Such a trail would be formed by hot liquid thrown off by the moving bubble (see also below).

11. It has been inferred $(\$ 4)$ that a bubble of vapour cannot remain of constant size except under continuous evaporation and condensation of liquid. This can be demonstrated in the following way :-

When the amplitude of the oscillations of the bubble is continuously reduced in the way described in $\$ 8$, the bubble frequently becomes so small that it comes to rest before it disappears, by adhering to a minute thread or roughness (visible through a microscope) upon the wire. It is generally possible to adjust the current so that this stationary bubble remains of constant size instead of gradually disappearing. It is then easy to observe that a stream of hot liquid is rising from the side of the bubble remote from the wire.

It is probable that in this case the film of liquid between the bubble and the wire is excessively thin. The central part of the film may even disappear. Liquid will be approaching it continuously owing to capillarity, but may be volatilising at such a rate as to keep the film interrupted at its centre. The wire will tend to become very hot at this point, for it must be remembered that the current through the wire (when a bubble is present) is always such that the wire would become incandescent if the liquid were removed. The evaporation into the bubble near the wire is compensated by an equal condensation at the remote part of the bubble, the temperature being kept below the boiling point here by the mixing of the condensed vapour with the surrounding cooler liquid. 'This process produces the rising stream.

It will be noticed that the displacement of such a bubble in either direction along the wire would bring fresh liquid over a very hot part of the wire. The sudden heating and expansion of this liquid would provide an impetus tending to move the bubble very rapidly in the direction of displacement.

12. An exhaustive consideration of all possible causes of 
motion of the bubble has not, of course, been attempted. It is possible that the explanations put forward above, which are reminiscent of those given in the case of the Trevelyan Rocker, are incomplete. For instance, differences of surface tension due to difference of temperature between the two sides of the bubble may produce an effect which has been disregarded. And it may easily happen that a better way of explaining the phenomena will occur to some observer tempted, by their interesting and possibly instructive nature, to take the small amount of trouble required to produce them.

\section{ADDED OCT. 21, 1915.}

Since writing the above account of experiments performed in May of last year,* it has occurred to me that it might be instructive, in experiments like those of $\$ 10$, to obtain some jdea of the temperature of the wire in the different cases. Some additional observations, with a wire similar to that previously used, have therefore been made. The result: are tabulated below : -

\begin{tabular}{|c|c|c|c|}
\hline Amperes. & Volts. & Resistauce. & 'Temperature. \\
\hline $2 \cdot 85$ & $\begin{array}{l}2 \cdot 20 \text { (no bubble) } \\
2 \cdot 17 \text { (bubble) }\end{array}$ & $\begin{array}{l}0.772 \\
0.761\end{array}$ & $\begin{array}{c}104 \cdot 0^{\circ} \mathrm{C} \\
99.0^{\circ}\end{array}$ \\
\hline $2 \cdot 9 \tilde{5}$ & $\begin{array}{l}2.31 \text { (no bublile) } \\
2 \cdot 26 \text { (bubble) }\end{array}$ & $\begin{array}{l}0.783 \\
0.766\end{array}$ & $\begin{array}{l}100 \cdot 5^{\circ} \\
101 \cdot 5^{\circ}\end{array}$ \\
\hline
\end{tabular}

The numbers in the first and second columns are currents and corresponding voltages (mean values) obtained as in $\$ 10$. The numbers in the third column are the resistances obtained by dividing the volts by the amperes in each case.

To obtain the numbers in the fouth column, the liquid was heated by an external source to about $1: 30^{\circ} \mathrm{C}$. It was then allowed to cool slowly, being liept stirred meanwhile. The resistance and temperature of the wire were measured from time to time. The numbers in the fourth column give the temperatures at which the wire had the resistances shown in the third.

From these data it is seen that, both with and without the bubble, the average temperature of the wire is $50^{\circ} \mathrm{C}$, or more below the boiling point of the turpentine (approx. $160^{\circ} \mathrm{C}$.). The temperature of the bubble itself cannot be below the boiling point. It is thus always much hotter than the liquid which it

$$
\text { *See "Bulletin," Phys. Soc., 1914., p. } 44 .
$$


displaces during its motion. But its temperature is maintained by the wire. Therefore that part of the wire upon which the bubble bears at any instant is much hotter than the rest. It is easy to see how this may occur. The thin film sandwiched between the hot bubble and the wire, no longer able to transfer the heat supplied by the wire to surrounding colder layers, is practically instantaneously raised to the boiling point.* The bubble is maintained by evaporation from it.

These experiments show, perhaps more explicitly than any of the others, where the force propelling the bubble originates.

\section{ABSTRACT.}

The first of these experiments was due to Mr. Addenbrooke who, using a 100-volt lamp filled with paraffin oil (after removing the tip) as a convenient high resistance in a 200 -volt circuit, noticed that some of the many bubbles forming on the filament behaved in a curious way. Instead of rising at once to the surface from the point at which therformed they ran down the legs of the filament, against gravity, and then escaped at the leading-in wires.

Dr. Smith, led to repeat this experiment by Mr. Crawley, discovered another, more striking, phenomenon. Placing the 100-volt lamp in a 100-volt eircuit in series with a variable resistance (conveniently a water-trough) it was found possible, by momentarily cutting out most of the resistance, to obtain a single bubble upon the wire. The be. haviour of such a bubble is very interesting to watch. Instead of escaping at either terminal, as in Mr. Addenbrooke's experiment, it travels backwards and forwards between the two, "looping the loops" of the filament in a fascinating way during every journey.

The peculiarities of this phenomenon, which can be obtained with either direet or alternating supply, have been analysed by examining the size and motion of the bubble under various conditions and also by using filaments of different materials and liquids of different boiling points.

It was shown, from the experiments, that a rapid fall of temperature from the wire through the liquid, in the region through which the bubble moves, is an essential condition of the phenomenon, and also, from theoretical considerations, how this condition can be used to explain why the bubble moves in the manner described.

* In the experiment by Mr. Moss, referred to in $\$ 2$, some of the air bubbles being too large did not attach themselves to the wire as intended. Passing across the wire, they rose to the surface of the oil and, bursting there, discharged minute clouds of smoke. The short time during which a film cxisted upon the wire, while the bubble passed across it, had sufficed to enable the current to heat the film to decomposition. 